\title{
Resonance production in high energy collisions from small to big systems
}

\author{
K. Werner ${ }^{(a)}$, A. G. Knospe ${ }^{(b)}$, C. Markert ${ }^{(c)}$, B. Guiot ${ }^{(a, d)}$, lu.Karpenko ${ }^{(a, e, f)}$, T. Pierog ${ }^{(g)}$, G. \\ Sophys $^{(a)}$, M. Stefaniak ${ }^{(a, h)}$, M. Bleicher ${ }^{(i, j)}$, J. Steinheimer ${ }^{(i)}$
}

(a) SUBATECH, University of Nantes - IN2P3/CNRS - IMT Atlantique, Nantes, France

(b) University of Houston, Houston, USA

(c) University of Texas, Austin, USA

(d) Universidad Tecnica Federico Santa Maria y Centro CientificoTecnologico de Valparaiso, Valparaiso, Chile

(e) Bogolyubov Institute for Theoretical Physics, Kiev 143, 03680, Ukraine

( $f$ ) INFN - Sezione di Firenze, Via G. Sansone 1, I-50019 Sesto Fiorentino (Firenze), Italy

(g) Karlsruhe Inst. of Technology, KIT, Campus North, Inst. f. Kernphysik, Germany

(h) Warsaw University of Technology, Warsaw, Poland

(i) FIAS, Johann Wolfgang Goethe Universitaet, Frankfurt am Main, Germany

(j) GSI Helmholtzzentrum, Planckstr. 1, 64291 Darmstadt

\begin{abstract}
The aim of this paper is to understand resonance production (and more generally particle production) for different collision systems, namely proton-proton ( $p p$ ), proton-nucleus (pA), and nucleus-nucleus (AA) scattering at the LHC. We will investigate in particular particle yields and ratios versus multiplicity, using the same multiplicity definition for the three different systems, in order to analyse in a compact way the evolution of particle production with the system size and the origin of a very different system size dependence of the different particles.
\end{abstract}

The main goal of heavy ion physics at very high energies is the proof of existence of the creation of a quark-gluon plasma, and the study of the properties of this exotic state, by analysing the final state of many thousands of produced hadrons. Unfortunately, many of these final state particles are not directly coming from the decay of the plasma, but they are produced or at least affected by the hadronic cascade, the last stage of the collision before particles freeze out and freely move to the detectors. Here resonance studies come into play: There is a large number of resonances at our disposal, having very different lifetimes, from $1 \mathrm{fm} / \mathrm{c}$ to several tens of $\mathrm{fm} / \mathrm{c}$, which means that these particles decay with varying probabilities in the hadronic stage, and provide therefore valuable information about the latter one.

We extended this analysis, to also consider small collision systems (pp and pA), in addition to AA. We also consider not only resonances, but also stable particles, providing useful additional information (the lifetime is not the only relevant parameter).

Our tool to analyse particle production is the EPOS model. EPOS3 [1] is a universal model in the sense that for pp, $\mathrm{pA}$, and AA collisions, the same procedure applies, based on several elements: 
(a)

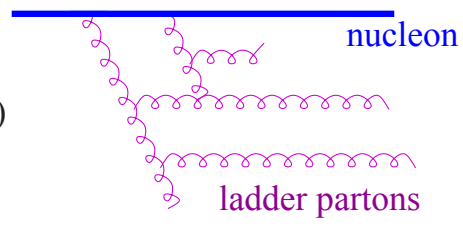

(b)

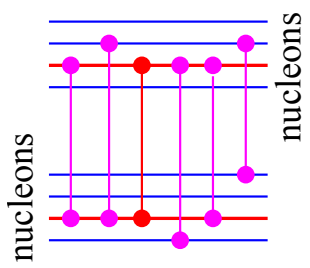

Figure 1. (Color online) (a) Non-linear effect: ladder fusion. (b) Pomerons connected to a given (red) Pomeron

Initial state. A Gribov-Regge multiple scattering approach is employed ("Parton-Based GribovRegge Theory" PBGRT [2]), where the elementary object (by definition called Pomeron) is a DGLAP parton ladder. Starting from a multi-Pomeron structure for the elastic scattering S-matrix, one then uses cutting rules to obtain (partial) cross sections of inelastic processes, by employing Markov chain techniques.

Non-linear effects. High parton density effects like gluon fusion during the parton evolution (see fig. 1(a)) are taken into account by using a dynamical saturation scale for each Pomeron, of the form $Q_{s}=Q_{s}\left(N_{\text {Pom }}, \hat{s}\right)$, depending on the number of Pomerons $N_{\text {Pom }}$ connected the Pomeron in question (see fig. 1(b)), and its energy $\hat{s}$. The functional form of $Q_{s}$ is obtained from fitting to the energy dependence of basic cross sections and the multiplicity dependence of particle production in $\mathrm{pp}$ at very high transverse momenta.

Core-corona approach. The parton ladders corresponding to Pomerons are treated as classical relativistic (kinky) strings. So in general, we have a large number of (partly overlapping) strings. Based on the momenta and the density of string segments, one separates at some early proper time $\tau_{0}$ the core (going to be treated as fluid) from the corona (escaping hadrons, including jet hadrons). The corecorona procedure has been first described in [3], a more recent discussion is found in [1]. The corresponding energy-momentum tensor of the core part is transformed into an equilibrium one, needed to start the hydrodynamical evolution. This is based on the hypothesis that equilibration happens rapidly and affects essentially the space components of the energy-momentum tensor.

Viscous hydrodynamic expansion. Starting from the initial proper time $\tau_{0}$, the core part of the system evolves according to the equations of relativistic viscous hydrodynamics [1, 4], where we use presently $\eta / s=0.08$. A cross-over equation-of-state is used, compatible with lattice QCD $[5,6]$. The "core-matter" hadronizes on some hyper-surface defined by a constant temperature $T_{H}$, where a so-called Cooper-Frye procedure is employed, using equilibrium hadron distributions, see [6].

Final state hadronic cascade. After hadronization, there occur still hadron-hadron rescatterings, realized via UrQMD [7].

The above procedure is employed for each event (event-by-event procedure).

We will discuss particle production as a function of the average multiplicity per eta interval $\langle d n / d \eta(0)\rangle$ at central pseudorapidity $(\eta=0)$, whereas forward pseudorapidity intervals are used to define the different multiplicity classes. This is the same procedure as used by the ALICE experiment. In the following $\langle d n / d \eta(0)\rangle$ is simply referred to as "multiplicity".

Crucial for the following discussion is the fact that the core-corona separation behaves quite differently for small and big systems (although we employ the same procedure), see fig. 2. Whereas 


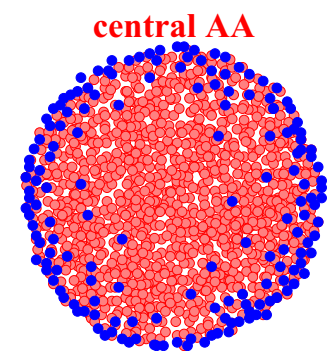

peripheral AA

high mult pp,pA

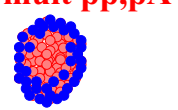

low mult pp

$\leftrightarrow$

Figure 2. (Color online) Core (red) and corona (blue) in a plane perpendicular to the beam axis, for big (left), small (right) and intermediate (middle) systems.

for very large multiplicity (central AA) most of the matter is core, with small corona contributions at the surface (or some high pt segments further inside), we get at very low multiplicity (in pp) only corona : the system is not dense enough to form a core. High multiplicity pp and pA, or peripheral AA collisions are in between, both core and corona being important.

Another way of seeing the multiplicity dependence of the core-corona separation is shown in fig. 3(a), where we plot the pion yield versus multiplicity, for only core particles ("core", green dasheddotted), the contribution from core + corona ("co-co", particles from core and corona, yellow dashed), both contributions from EPOS simulation without hadronic cascade. The ratio "core" over "co-co" would be the relative core fraction, which increases continously from zero (at small multiplicity) to unity (at large multiplicity). We also plot the "full" contribution, referring to all particles, for a simulation with hadronic cascade (A summary of color, line, and symbol codes is found in table 1). In all cases we plot curves for the three systems: pp, pA, and AA. There are substantial overlap regions, where different systems contribute, but we observe unique curves, no system size dependence. We also observe that the hadronic cascade has no effect on the pion production ("co-co" and "full" gives identical results).

In fig. 3(b), we plot a quantity relevant for resonance decays in the hadronic stage: The lifetime of this phase, defined as the difference of the average formation time of particles for simulations with and

\begin{tabular}{|c|c|c|c|}
\hline core & green dashed-dotted & particle from the core only & no hadronic cascade \\
\hline corona & blue dotted & particles from corona only & no hadronic cascade \\
\hline co-co & yellow dashed & particles from core and corona & no hadronic cascade \\
\hline full & red full & all particles & with hadronic cascade \\
\hline & blue triangles & particles from pure string decay & no cascade, no hydro \\
\hline \hline & thin lines & pp simulation & \\
\hline & intermediate lines & $\mathrm{pA}(\mathrm{pPb})$ simulation & \\
\hline & thick lines & $\mathrm{AA}(\mathrm{PbPb})$ simulation & \\
\hline \hline & open circles & $\mathrm{pp} \mathrm{data}$ & \\
\hline & open squares & $\mathrm{pA}(\mathrm{pPb})$ data & \\
\hline & open stars & $\mathrm{AA}(\mathrm{PbPb})$ data & \\
\hline
\end{tabular}

Table 1. Color, line, and symbol codes used for the plots in this paper, to accommodate the different contributions and the different systems. 
(a)

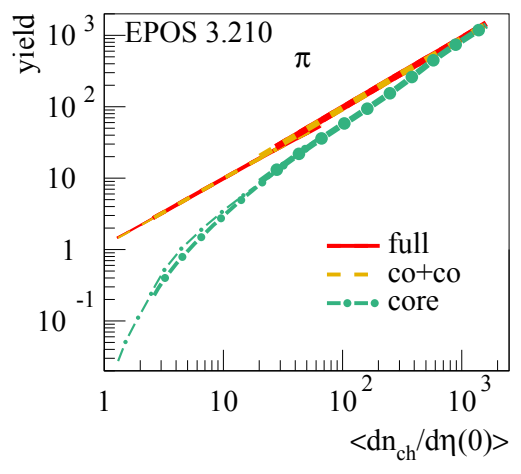

(b)

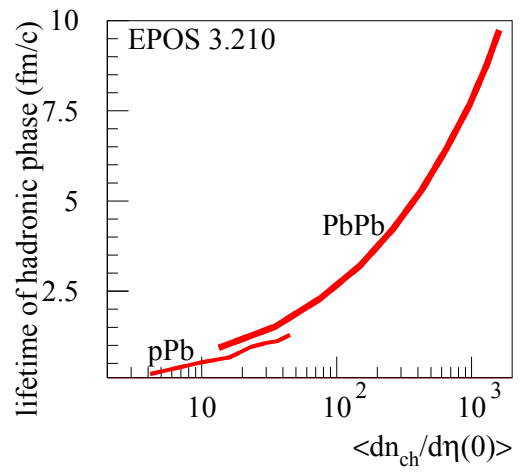

Figure 3. (Color online) (a) Pion yield versus multiplicity. We compare different contributions (core, core plus corona (co-co) and the the full contribution (the latter on including the final state hadronic cascade) for different systems: pp (thin lines), AA (thick lines), and pA (intermediate lines). (A summary of color, line, and symbol codes is found in table 1) (b) Lifetimes of the hadronic phase versus multiplicity.

without hadronic cascade. The bigger the lifetime, the bigger the probablility of a resonance decay inside this phase. The lifetime increases continuously from less than a $\mathrm{fm} / \mathrm{c}$ to $10 \mathrm{fm} / \mathrm{c}$ for big systems.

In the following we will investigate the multiplicity dependence (for pp, $\mathrm{pA}, \mathrm{AA}$ ) of particle ratios to pions, for the different contributions from EPOS simulations, compared to data from ALICE [817]. We always refer to the color, line, and symbol codes of table 1.

In fig. 4(a), we plot the kaon to pion ratio versus multiplicity, for different contributions from the EPOS simulations, for different systems (pp, pA, AA), see table 1 for color, line, and symbol codes. Despite a large overlap for the different systems, we observe universal curves, for all contributions. The core contribution is completely flat, as expected since the particle ratios only depend on the properties of the fluid at freeze out, taken to be the same in all systems. Also the corona contribution (from string segments which escape the fluid) is essentially flat, but with some deviation from pure string fragmentation (blue triangles). But at large multiplicity the corona contribution is small, so we have essentially two flat curves, from core and corona, where here in case of kaons the core contribution is roughly a factor of 2 above the corona. From fig. 3(a), we know that the relative core weight increases continuously from zero to unity, which explains the increase of the core+corona (co-co) contribution which interpolates between the corona level at small multiplicity to the core level at high multiplicity. Considering finally the "full" contribution (including the final state hadronic cascade), we see no difference compared to the case without cascade (co-co), both curves reproducing the experimental data. In fig. 4(b), we consider $\phi$ mesons, and we observe almost the same behavior as seen for the kaons, a continuous increase of roughly a factor of 2 from corona to core level, and little effect of the hadronic cascade. Due to the long lifetime of $46.2 \mathrm{fm} / \mathrm{c}$ only few $\phi$ mesons decay in the hadronic phase.

The situation is different for $\boldsymbol{\Omega}$ baryons, see fig. 4(c), since here core and corona curves are separated by about a factor of 10 , leading to a strong increase of the core+corona (co-co) contribution of a factor of 10 with multiplicity. The full curve is slightly reduced at high multiplicity compared 
(a)

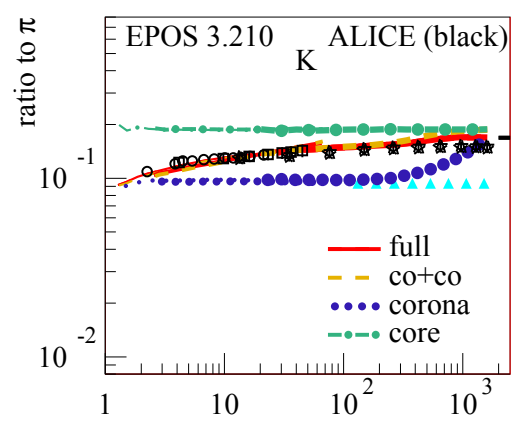

(c)

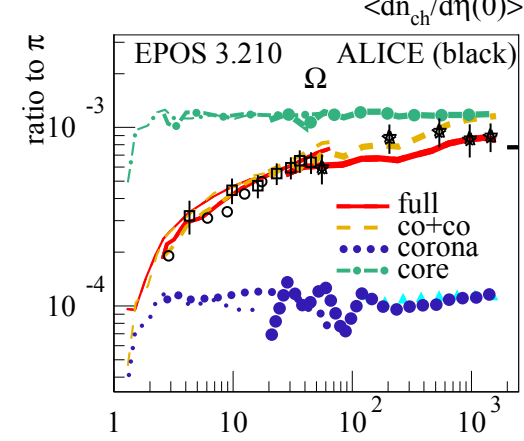

(e)

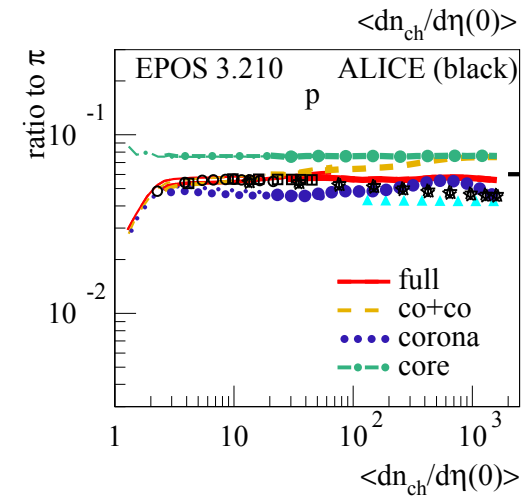

(b)

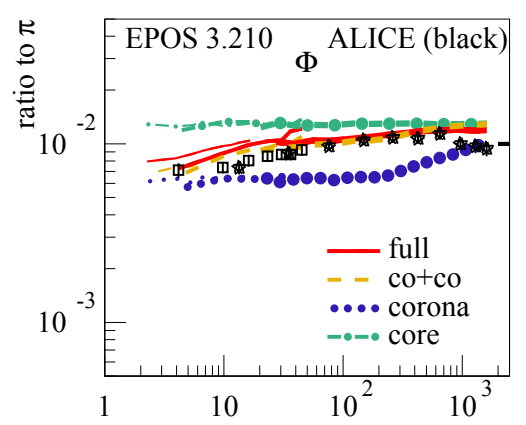

(d)

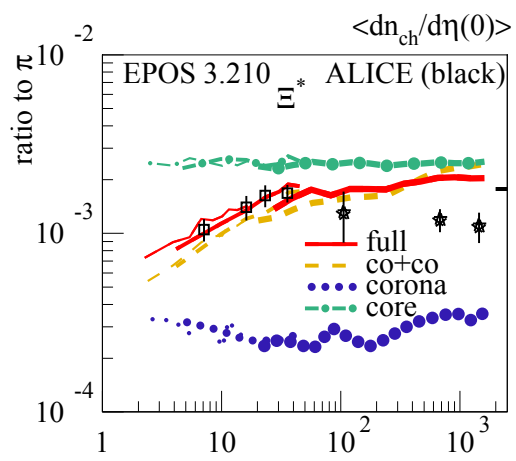

(f)

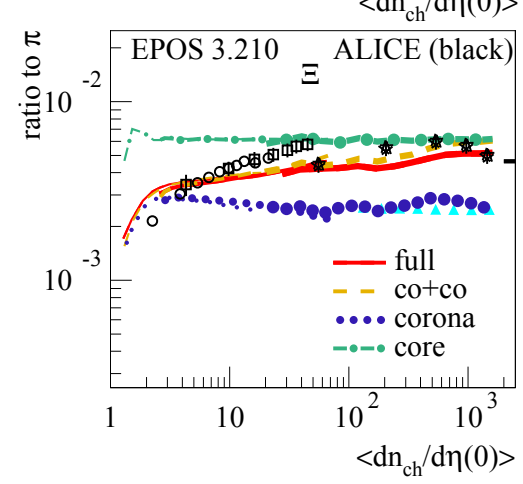

Figure 4. (Color online) Particle to pion ratio for different particle species versus multiplicity, for different contributions from the EPOS simulations, for different systems (pp, pA, AA). We also plot ALICE data from [8-20]. See table 1 for color, line, and symbol codes.

to co-co, due to hadronic final state interactions (baryon-antibaryon annihilation). We observe very similar results for the $\Xi^{*}$ resonance (lifetime $21.7 \mathrm{fm} / \mathrm{c}$ ), but some discrepancy compared to the data.

Concerning protons, we see in fig. 4(e) also first an increase of the co-co curve, form the corona level to the core level, and again (as for the Omega resonance) a reduction at high multiplicity when including final state hadronic rescattering, coming from baryon-antibaryon annihilation. We also plot $\boldsymbol{\Xi}$ baryons in fig. 4(f), showing a significant increase with multiplicity.

In fig. 5(a), we consider the kaon resonance $\boldsymbol{K}_{\mathbf{0}}^{*}$. Here core, corona, and as a consequence also core+corona (co-co) are identical (by accident). Interesting: The full results (including the final state hadronic cascade) is considerably lower than the results without cascade (co-co). This is due to 
(a)

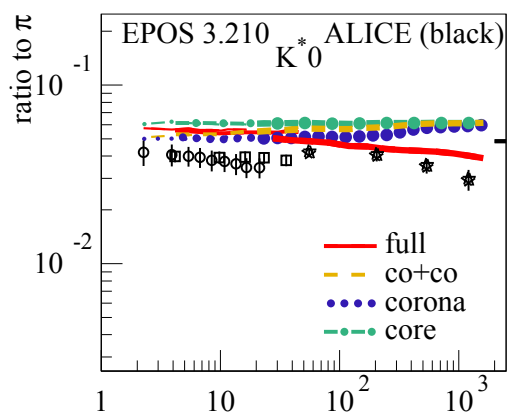

(c)

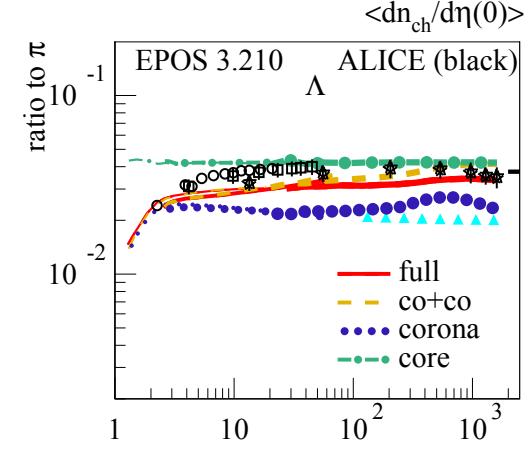

(e)

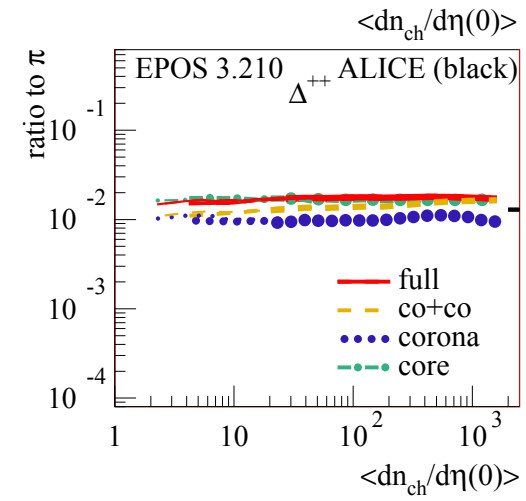

(b)

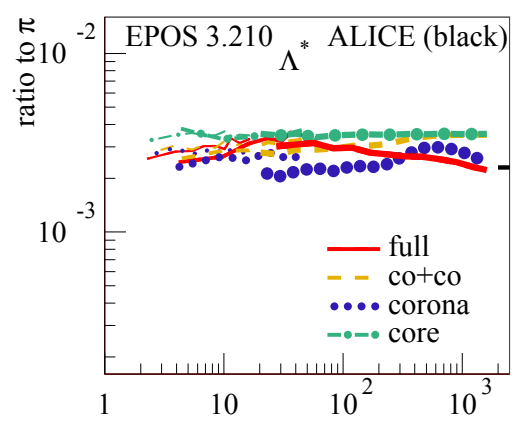

(d)

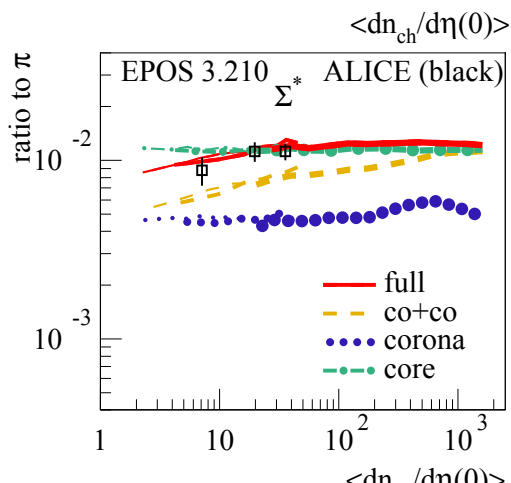

(f)

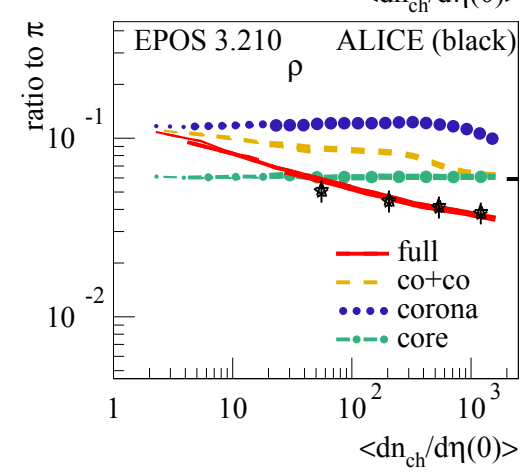

Figure 5. (Color online) Same as fig 4 , for other particle species.

the short lifetime $(4.2 \mathrm{fm} / \mathrm{c})$, which makes the particles decay within the hadronic stage. Some of the decay products rescatter (and change momenta) and thus prevent the kaon resonances to be reconstructed via their decay products. So contrary to the cases discussed earlier, we observe here a monotonically decreasing curve, as observed in the data for large multiplicities. The data show some discontinuity at low multiplicity, contrary to what we observe in the simulation. Newer ALICE results show as well a continuous behavior. Also the Lambda resonance $\boldsymbol{\Lambda}^{*}$ shows in $5(\mathrm{~b})$ a significant decrease of the "full" contribution at high multiplicity, partly due to decays in the hadronic stage (lifetime $12.6 \mathrm{fm} / \mathrm{c}$ ) and baryon-antibaryon annihilation. But one has to be careful not to draw wrong conclusions. The lifetime is relatively large, so the effect of the decay cannot be very big compared to Lambdas. On the other hand, it seems that the corona and the core level are closer to each other 
compared to the Lambda (see fig. 5(c)), so the core+corona curve increases less, and therefore the reduction due to annihilation (present in both $\Lambda$ and $\Lambda^{*}$ ) provides a bigger decrease in the case of $\Lambda^{*}$.

The $\Sigma^{*}$ resonance, shown in fig. 5(d), shows also the "usual" monotonic increase of the core+corona contribution, but here we get a strong increase at low multiplicity but little change at high multiplicity, when considering the "full" contribution including the hadronic cascade. We have actually two competing processes: The lifetime is relatively short $(5 \mathrm{fm} / \mathrm{c})$, so there should be some reduction due to decay at high multiplicity, which is not visible because it is compensated by an increase due to resonance creation in the hadronic stage. Whereas the reduction due to decay is only possible for sufficiently big systems (at high multiplicity), resonance creating is possible at any multiplicity, and is therefore visible at small multiplicities. The $\Delta^{++}$resonance (lifetime $1.7 \mathrm{fm} / \mathrm{c}$ ), considered in fig. 5(e), shows a similar behavior, on a smaller scale.

An exceptional case is the $\rho$ resonance, shown in fig. 5(f), since here the core level is below the corona one (this is the only case, it happens because $\rho$ production is very frequent in the string breaking procedure), and therefore the core+corona contribution decreases with multiplicity. Considering the full contribution (including the hadronic cascade), there is an additional reduction, such that altogether there is a very strong decrease of the $\rho$ over pion yield with multiplicity.

To summarize, we have shown that the study of the multiplicity dependence of particle ratios for different collision systems ( $\mathrm{pp}, \mathrm{pA}, \mathrm{AA}$ ) provides a wealth of information concerning the production mecanism of particles and about the properties of the medium having produced or affected these particles.

\section{Acknowledgements}

This research was carried out within the scope of the GDRE (European Research Group) "Heavy ions at ultrarelativistic energies", and was partially supported by COST Action CA15213 (THOR). This work was supported by U.S. Department of Energy Office of Science under contract number DE-SC0013391. The authors acknowledge the Texas Advanced Computing Center (TACC) at the University of Texas at Austin for providing computing resources that have contributed to the research results reported within this paper. URL: http://www.tacc.utexas.edu. B. G. gratefully acknowledges generous support from Chilean FONDECYT grants 3160493 and Proyecto Basal FB0821.

\section{References}

[1] K. Werner, B. Guiot, I. Karpenko, and T. Pierog, Phys.Rev. C89 (2014) 064903, arXiv: 1312.1233.

[2] H. J. Drescher, M. Hladik, S. Ostapchenko, T. Pierog and K. Werner, Phys. Rept. 350, 93, 2001

[3] K. Werner, Phys. Rev. Lett. 98, 152301 (2007)

[4] Iu. Karpenko, P. Huovinen, M. Bleicher, arXiv:1312.4160

[5] S. Borsanyi et al., JHEP 1011 (2010) 077, arXiv:1007.2580

[6] K. Werner, Iu. Karpenko, T. Pierog, M. Bleicher, K. Mikhailov, arXiv:1010.0400, Phys. Rev. C 83, 044915 (2011)

[7] M. Bleicher et al., J. Phys. G25 (1999) 1859; H. Petersen, J. Steinheimer, G. Burau, M. Bleicher and H. Stocker, Phys. Rev. C78 (2008) 044901

[8] ALICE Collaboration, Phys. Rev. Lett. 106032301 (2011), Phys. Rev. C 88044910 (2013)

[9] ALICE Collaboration, Phys. Rev. Lett. 111222301 (2013)

[10] ALICE Collaboration, Phys. Lett. B 758 389-401 (2016)

[11] ALICE Collaboration, Phys. Lett. B 728 25-38 (2014) 
[12] ALICE Collaboration, Eur. Phys. J. C 76245 (2016)

[13] ALICE Collaboration, Phys. Lett. B 712309 (2012)

[14] ALICE Collaboration, Phys. Lett. B 728 216-227 (2014)

[15] ALICE Collaboration, Phys. Rev. C 91024609 (2015)

[16] ALICE Collaboration, Nature Physics 13 (2017) 535-539

[17] digitized from plenary talk of Rafael Derradi de Souza (for the ALICE Collaboration), SQM2016

[18] derived from talk of Jihye Song (for the ALICE Collaboration) at Quark Matter 2017

[19] digitized from A. G. Knospe (for the ALICE Collaboration) at SQM 2016

[20] ALICE, Eur. Phys. J. C 77 (2017) 389 